\title{
ANALYTICAL AND NUMERICAL COMPUTATION OF ELECTRICAL RESISTANCE IN A LOW CURRENT MULTI-SPOTS METALLIC CONTACT
}

\author{
Gideon Gwanzuwang DANKAT ${ }^{1}$, Alin Alexandru DOBRE ${ }^{1}$, and \\ Laurentiu Marius DUMITRAN ${ }^{1}$, \\ ${ }^{1}$ University Politehnica of Bucharest, Laboratory of Electrical Materials \\ gdankat@elmat.pub.ro ${ }^{1}$, alin.dobre@upb.ro and dumitran@elmat.pub.ro
}

\begin{abstract}
The problem concerning the specific resistance of electrical contacts is still of special importance especially in high complexity electrical systems or critical operation devices such as those used in the automotive industry. A huge number of studies have been conducted over time in order to develop easy models that allow the calculation of the contact resistance at the interface between two conductors taking into account the discontinuity of the contact surface. This paper presents a study on the computation of the contact resistance of two metal conductors with analytical relations and, respectively, using numerical computation models. The aim of this paper is to examine the differences between the results obtained using analytical models and numerical model based on finite element method (FEM) in COMSOL Multiphysics.
\end{abstract}

\section{INTRODUCTION}

Electric connectors are one of the most important components in electronics and automotive systems. Faults in automotive sensor connectors have been linked to degraded connectors and high contact resistance [1]. Measuring the contact resistance determines the condition and the characteristics of a connector. In machinery assembly, evaluating the reliability and tightness of a contact can be done using the contact resistance of metals contact surface. The conduction characteristic of the contact surface is associated with contact resistance. The larger area and the less impurity the metal surfaces have, the better the conductivity and the lower resistance are, and vice versa [2].

The features of contact surface, rough and unclean, form the constriction resistance and film resistance of rivets. Contact resistance and contact temperature rise coexist for a long time and are influenced by each other. Abnormal increase of contact resistance and contact temperature can induce electrical contact welding, short circuit, fire casualty etc. The correlative factors include contact force, current, material physical properties, metal oxides and interface characteristics. Contamination on the surface of contact leads to an increase in electrical resistance which can cause failure in contact applications. The constriction of the contact area can be seen in (Fig. 1). Contact resistance measurements aid in identifying resistance elements that have increased above acceptable values. The operation of electrical equipment relies on the controlled flow of current within designated parameters of the given piece of equipment. Contact resistance measurements are expected to avert long term damage to existing equipment and to reduce energy wasted as heat. They indicate any restrictions in the current flow that might hinder a machine from generating/achieving its full power or allow insufficient current to flow to activate protective devices in the case of a fault [3].

The aim of this study is to compare, using analytical approach and numerical simulation, the contact resistance in a simple case of two metallic discs in contact through multiple regular circular spots. Despite all the assumptions considered in the analytical models, the present study aims to explore the limits of numerical calculation of the contact resistance in the case of a fairly simple geometry. Moreover, the paper also examined the influence of the size of contact spots on the value of contact resistance. 


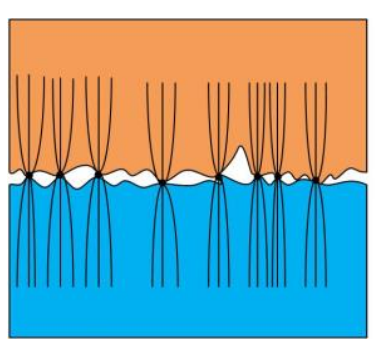

a)

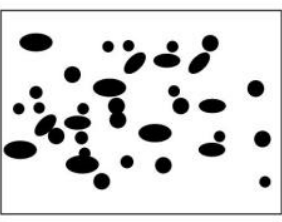

b)

Fig.1. Diagram of a bulk electrical interface showing: (a) the constriction of the current lines, (b) the contact spots. [4]

\section{ANALYTICAL MODELS FOR CONTACT RESISTANCE.}

Numerous studies have been published on electrical contact and contact resistance; it is still a major topic of interest in the engineering field today [4-6]. In most studies regarding electric contact and contact resistance, reference was made to Holm's and Greenwood's work on the analytical model for the calculation of contact resistance. In this paper, the Holm's and Greenwood's equation will be used to calculate analytically the value of contact resistance based on a simplified model of two metal discs coming in contact through multiple spots.

\subsection{Holm's model}

Holm described the basics of his theory by considering two cylinders, $C_{1}$ and $C_{2}$ which are in contact with each other. He denoted the apparent contact area as $A_{a}$ (see fig.2).

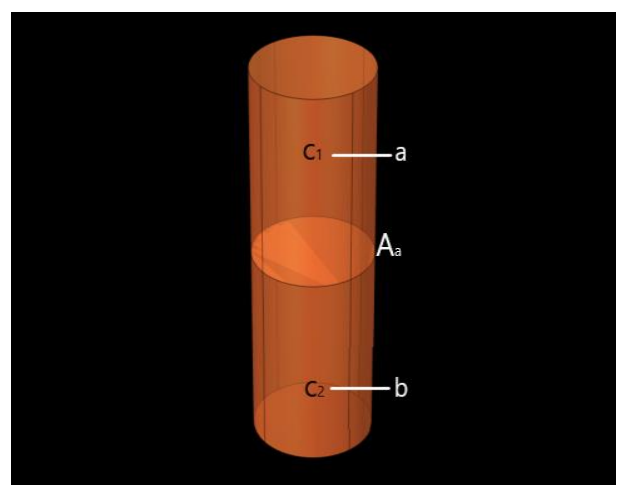

a)

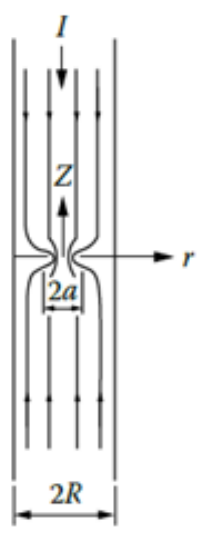

b)

Fig.2. Holm's contact resistance theory example: a) apparent area of contact $A_{a}$;

b) conductor cylinder of radius $R$ carrying circular constriction of radius $a$ [7].

Holm assumed that both faces of the cylinders are clean metallic but due to their rough surface nature, both cylinders come in contact via only a small portion of the apparent contact area $A_{a}$ denoted as $A_{c}$. As mentioned earlier, constriction resistance appears as a result of the current flow being constricted through $A_{c}$ which is made up of numerous spots ( $a$-spots) which depend on the size of the area and the shape of $A_{c}$. As 
the current flows through the contact, a voltage can be measured between points " $a$ " and " $b$ " and subsequently, the resistance between both surfaces. The total constriction resistance for one circular spot between two electrically conducting metallic cylinder (fig.3.) is given by the expression:

$$
R c=\frac{\rho}{2 a}
$$

where: $\rho$ - the resistivity of the conductor and $a$-radius of the constriction.

For cases where the bodies in contact differ in material properties i.e., having different resistivity $\rho_{1}$ and $\rho_{2}$, the constriction resistance becomes:

$$
R c=\frac{\rho_{1}+\rho_{2}}{4 a}
$$

Hypothesis in Holms theory:

- the channel through which the current flows is made of the same material (i.e. no presence of oxide films at the interface);

- the contact spot ( $a$-spot) has no thickness (i.e. no axial deviation in the direction of current flow);

- the conductors in contact are bulk conductors having dimensions transverse to the current flow to be infinite.

As mentioned earlier, most research studies or literature dealing with the properties of electrical contacts have often treated the contact spots in the form of a circular $a$-spot, but there are other forms of $a$-spots. For example, Nakamura et.al have done extended studies and derived formulas for the constriction resistance of $a$-spots with various shapes (triangular, square, hexagonal spots etc.) [8-11].

\subsection{Greenwood's theory}

Greenwood theory is based on a wider interpretation of Holms theory. In a paper published in 1966 by J.A Greenwood [12] he derived a formula for the calculation of constriction resistance. He considered a single cluster at the interface between two metallic electrodes consisting of $n$ micro-contacts (multiple $a$-spots) and it is smaller in size compared to the apparent contact area and the distance of that cluster to any other cluster. The metallic electrodes communicate through these spots with no interface film. He derived the formula by treating the current flow problem similar to that of an electrostatic charge distribution problem. Greenwood derived an equation for constriction resistance (3) based on multiple spots within a single cluster

$$
R_{G}=\frac{\rho}{2 \sum a_{i}}+\frac{\rho}{\pi}\left(\sum_{i \neq j} \frac{a_{i} a_{j}}{s_{i j}}\right) /\left(\sum a_{i}\right)^{2}
$$

where $\rho$ is the resistivity, $s_{i j}$ is the distance between the centers of spots $i$ and $j, a_{i}$ and $a_{j}$ are the radius of spots $i$ and $j$.

The first term $\frac{\rho}{2 \sum a_{i}}$ represents the resistance of all the spots in parallel. The second term $\frac{\rho}{\pi}\left(\sum_{i \neq j} \frac{a_{i} a_{j}}{s_{i j}}\right) /\left(\sum a_{i}\right)$; represents the resistance due to the interaction between all the spots. 
Greenwood did a further approximation of equation (3). When there is no correlation between the size of a given contact spot and its position, equation (3) becomes:

$$
R_{G 1}=\frac{\rho}{2 \sum a_{i}}+\frac{\rho}{\pi n^{2}} \sum_{i \neq j} \frac{1}{s_{i j}} .
$$

Equation (4) holds when all the contact spots are all the same size. Furthermore, the author confirmed Holm's earlier proposal that the total effect of a local constriction in a well filled cluster of contacts generates a resistance which can be calculated using eq. (5), by introducing the mean contact radius $\bar{a}$ and evaluating the summation of all the reciprocal distances involved in equation (4):

$$
R=\rho\left(\frac{1}{2 n a}+\frac{1}{2 \alpha}\right)
$$

where $\bar{a}$ is the mean $a$-spot radius, $n$ is the number of contact spots and $\alpha$ is the radius of the cluster. The hypothesis taken in Holm's theory still holds in Greenwood's theory. In 2001, L.Boyer published a paper on the generalizations of Greenwood's formula including interface films [13].

\section{NUMERICAL MODEL}

The investigated problem is that of a simple metallic disc (copper) in contact via multiple contact spots (fig.3). A low DC current of density $\boldsymbol{j}$ is injected by contact terminals and the distribution of electric field is calculated using the finite element method (FEM) and COMSOL Multiphysics software.

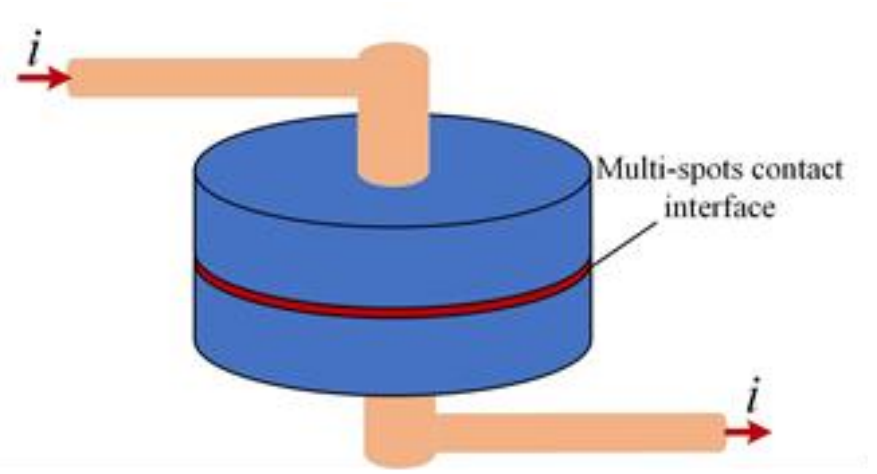

a)

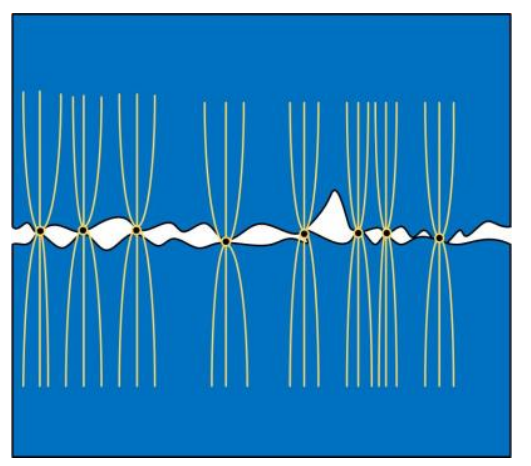

b)

Fig.3. (a) schematic view of metallic disc, (b) constriction of current lines flowing through the contact spots.

\subsection{Geometrical model}

The analysis investigates the contact of two metallic disks through multiple spots (total of 28 identical circular contact spots) with the apparent area of contact having a thin insulating layer of polyethylene with high resistivity (fig.4). Both metallic discs have the following dimensions: radius $\alpha=5 \mathrm{~mm}$ and thickness $h=1 \mathrm{~mm}$. The contact spots each have a radius $a=0.2 \mathrm{~mm}$. The insulating layer has a thickness of $30 \mu \mathrm{m}$. 


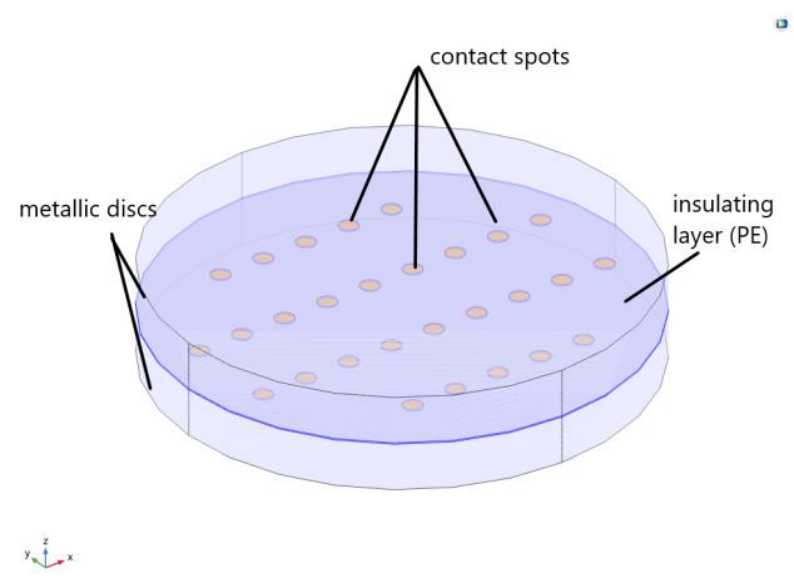

Fig.4. Geometrical model showing the contact spots.

\subsection{Mathematical model}

The numerical analysis consists of an electromagnetic problem and stationary electrokinetic regime with an imposed constant electric current of density $\boldsymbol{j}$ passing through the multiple contact spots. The fundamental equations governing the problem are: the electric charge conservation law (6), the electromagnetic induction law (7) and the electric conduction law (8)

$$
\begin{gathered}
\operatorname{div} \boldsymbol{J}=\mathbf{0}, \\
\operatorname{rot} \boldsymbol{E}=\mathbf{0}, \\
\boldsymbol{J}=\sigma \cdot \boldsymbol{E},
\end{gathered}
$$

where $\sigma[\mathrm{S} / \mathrm{m}]$ is the electric conductivity and $\boldsymbol{E}[\mathrm{V} / \mathrm{m}]$ is the electric field strength. $\boldsymbol{E}$ can be evaluated as a function of electric potential $V$ as

The boundary conditions are:

$$
\boldsymbol{E}=-\operatorname{grad} V
$$

- Continuity $\boldsymbol{n} \cdot\left(\boldsymbol{J}_{1}-\boldsymbol{J}_{2}\right)=0$. It specifies that the normal components of the electric currents are continuous across the interior boundaries of both metallic discs;

- Electric insulation $\boldsymbol{n} \cdot \boldsymbol{J}=0$. It is applied on all surfaces with the exception of the contact spots; it specifies that no current is flowing across the boundaries.

The equations system (6)-(9) was solved using Comsol Multiphysics and the finite element method. Fig.5 shows the discretization of the computational domain.
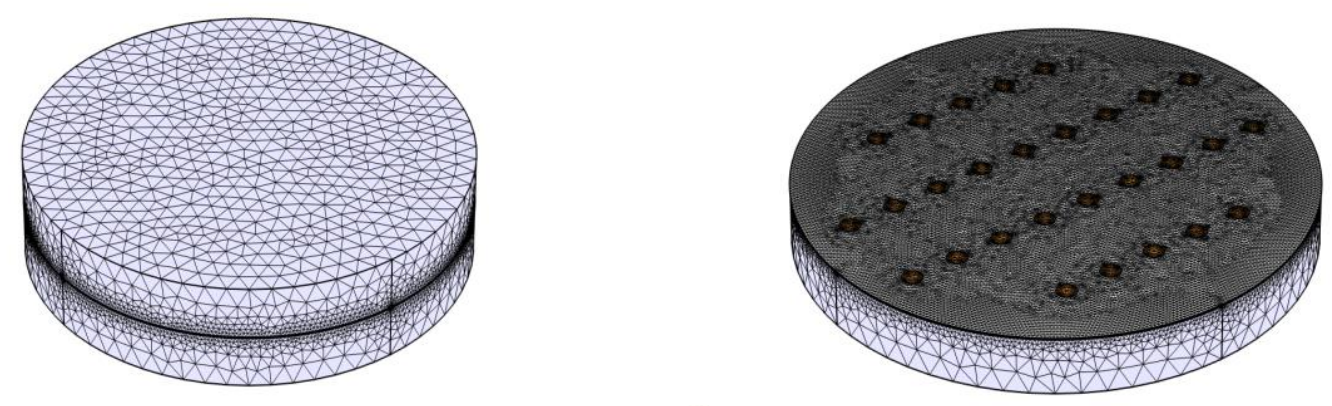

Fig.5. Discretization of the computational domain showing the contact spots. 


\section{RESULTS AND DISCUSSIONS}

Fig.6 shows the computed current flow lines for an applied current of $200 \mathrm{~mA}(J=$ $2.546 \cdot 10^{-3} \mathrm{~A} / \mathrm{mm}^{2}$ ).
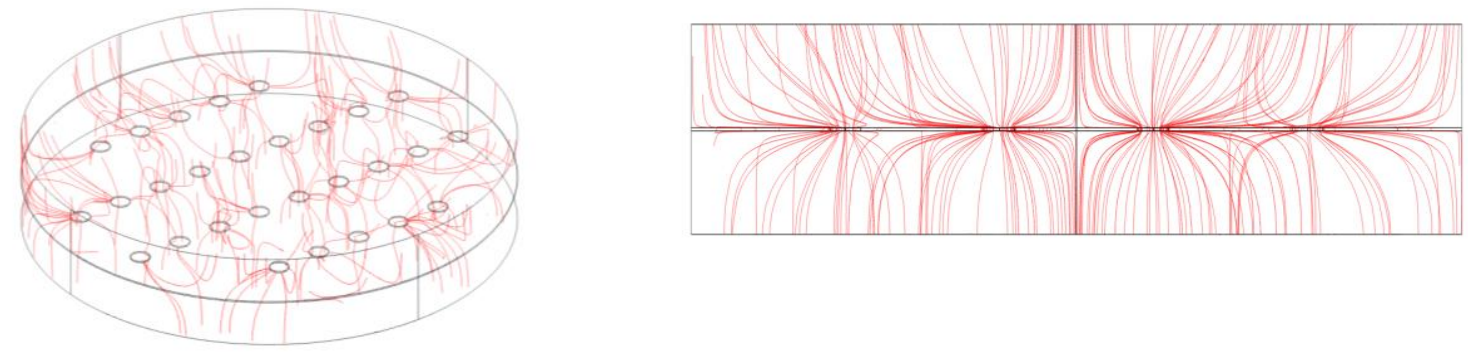

Fig.6. Constriction of current lines at the contact spots.

Fig.7 shows the computed voltage for an injected current of $200 \mathrm{~mA}\left(2.546 \cdot 10^{-3} \mathrm{~A} / \mathrm{mm}^{2}\right)$, $\sigma_{\mathrm{Cu}}=5.96 \cdot 10^{7} \mathrm{~S} / \mathrm{m}, \sigma_{\mathrm{PE}}=1 \cdot 10^{-17} \mathrm{~S} / \mathrm{m}$, and $T_{0}=20^{\circ} \mathrm{C}$. The result shows a maximum voltage of about $0.4 \mu \mathrm{V}$.
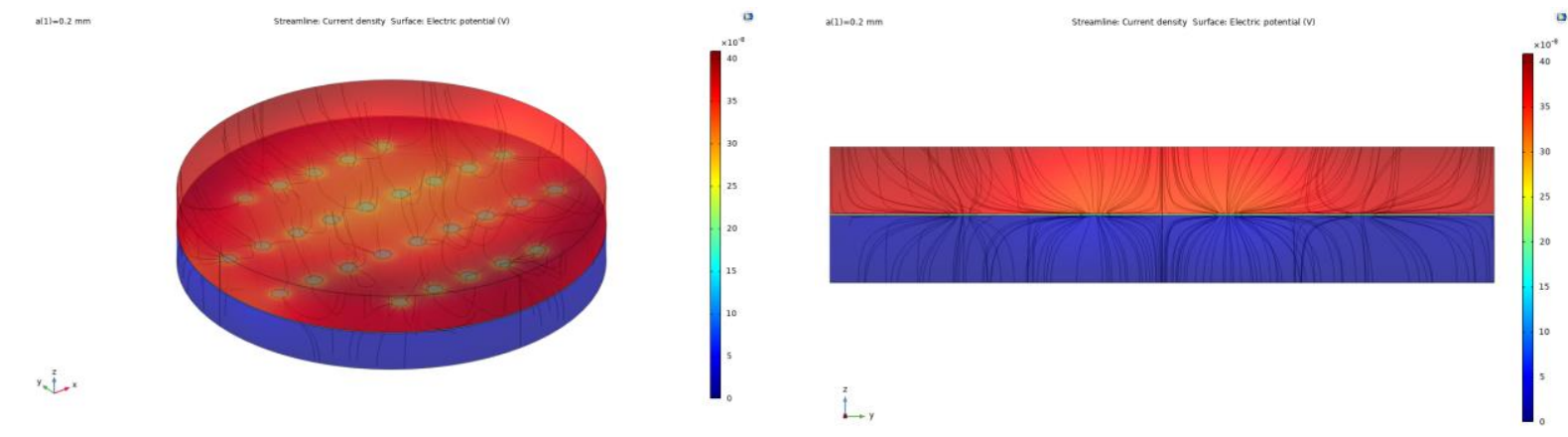

Fig.7. Computed voltage when the injected current is $200 \mathrm{~mA}$.

Figure. 8 shows the voltage drop for different radii values of the contact spots $(\mathrm{a}=0.1$ $\mathrm{mm}, 0.2 \mathrm{~mm}, 0.3 \mathrm{~mm}, 0.4 \mathrm{~mm}$ and $0.5 \mathrm{~mm})$. The results shows that when a $=0.1 \mathrm{~mm}$, the voltage drop is $V=6.5 \cdot 10^{-8}[\mathrm{~V}]$, when a $=0.2 \mathrm{~mm} ; V=2.62 \cdot 10^{-8}[\mathrm{~V}]$, when a $=0.3 \mathrm{~mm} ; V=$ $8.68 \cdot 10^{-8}[\mathrm{~V}]$, when a $=0.3 \mathrm{~mm} ; V=9.79 \cdot 10^{-8}[\mathrm{~V}]$ and when $\mathrm{a}=0.5 \mathrm{~mm} ; V=6.93 \cdot 10^{-8}[\mathrm{~V}]$,

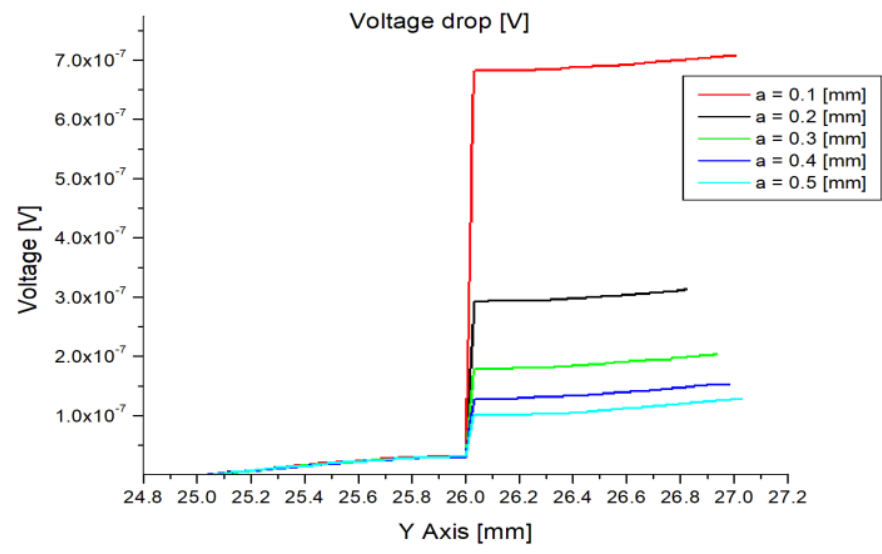

Fig.8. Voltage drop for different values of contact spots radius $(a=0.1 \mathrm{~mm}, 0.2 \mathrm{~mm}, 0.3 \mathrm{~mm}$, $0.4 \mathrm{~mm}$ and $0.5 \mathrm{~mm}$ ). 
Fig.9 shows a graphical representation of the calculated contact resistance values for both Holm's (1) and Greenwood's (4) equations and the value computed numerically. It can be seen that the contact resistance decreases as the contact spot radius increases in all three cases. The results shows that when the contact spot radius increases from $0.1 \mathrm{~mm}$ to $0.3 \mathrm{~mm}$, the contact resistance decreases from $3.07 \cdot 10^{-6} \Omega$ to $1.02 \cdot 10^{-6} \Omega$ (Holm), $4.76 \cdot 10^{-6} \Omega$ to $2.71 \cdot 10^{-6} \Omega$ (Greenwood) and $3.25 \cdot 10^{-6} \Omega$ to $4.34 \cdot 10^{-7} \Omega$ (numerical simulation). And when the contact radius increases to $0.5 \mathrm{~mm}$, the contact resistance becomes $6.14 \cdot 10^{-7} \Omega$ (Holm), $2.30 \cdot 10^{-6} \Omega$ (Greenwood) and $3.47 \cdot 10^{-7} \Omega$ (numerical simulation).

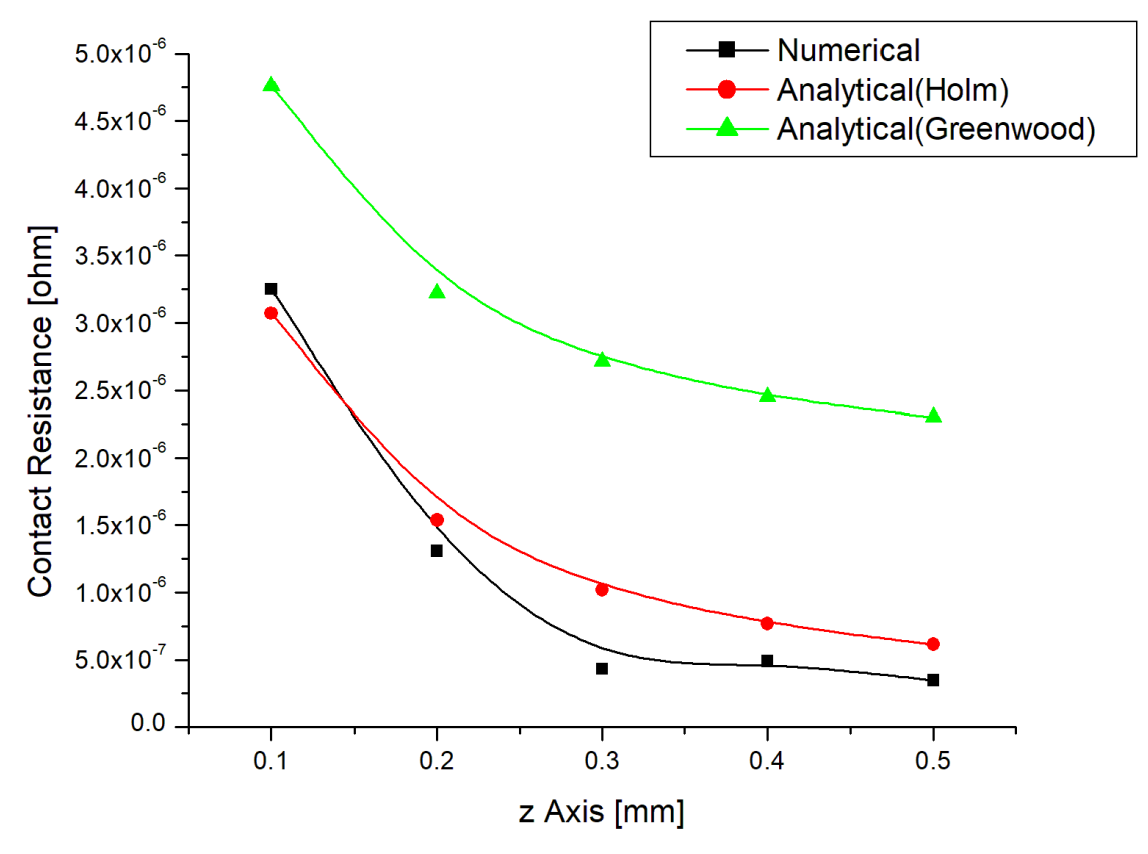

Fig.9. Contact resistance calculation showing analytical values Holm (1) and Greenwood (4) and numerical simulation values.

\section{CONCLUSION}

The present paper shows an analysis of the values of the contact resistance corresponding to an assembly of two copper disks that touch in 28 identical circular contact spots. The contact resistance was calculated using two analytical models in the first step, and with the help of a simple numerical model, in the second step. The calculations were performed keeping the number of spots constant and changing their radius between 0.1 to 0.5 $\mathrm{mm}$. The obtained results indicate that for both analytical models and any contact spot radius the contact resistance is higher than that obtained by numerical computation. Regardless of the used model and contact spot radius, the obtained values vary between $4.76 \cdot 10^{-6} \Omega$ (at a $=$ $0.1 \mathrm{~mm}$ (Greenwood)) and $3.47 \cdot 10^{-7} \Omega$ (at a $=0.5 \mathrm{~mm}$ (numerical)). Also, for a contact spot radius of $\mathrm{a}=0.1 \mathrm{~mm}$ the percentage difference for the value obtained numerically compared to Holm and Greenwood is $5 \%$ and $46 \%$ respectively. For contact spots of $0.3 \mathrm{~mm} 0.5 \mathrm{~mm}$, the value obtained for Holm is $135 \%$ and $76 \%$ more than the numerical value and the value obtained for Greenwood is 5 times more than the numerical value. 


\section{BIBLIOGRAPHY}

[1] J. Lim, H. Kim, J. K. Kim, S. J. Park, T. H. Lee and S. W. Yoon, "Numerical and Experimental Analysis of Potential Causes Degrading Contact Resistances and Forces of Sensor Connectors for Vehicles," in IEEE Access, vol. 7, pp. 126530-126538, 2019.

[2] Min LIU, "A New Method for Measuring Contact Resistance" Beijing Orient Institute of Measurement \& Test Chinese Academy of Space Technology.

[3] https://www.testequipmentdepot.com/megger/pdf/low-resistance-testing.pdf

[4] G. G. Dankat, A. A. Dobre and L. M. Dumitran, "Influence of Ageing on Electrothermal Condition of Low Current Contact," 2021 12th International Symposium on Advanced Topics in Electrical Engineering (ATEE), 2021, pp. 1-6.

[5] J. Swingler, J.W. McBride, C. Maul, "The degradation of road-tested automotive connectors", IEEE Trans. Comp. Packag. Technol 23 (1), pp. 157-164, 2000.

[6] Wang Shujuan, Hu Fang, Su Bonan and Zhai Guofu, "Method for calculation of contact resistance and finite element simulation of contact temperature rise based on rough surface contact model," 26th International Conference on Electrical Contacts (ICEC 2012), Beijing, pp. 317-321, 2012.

[7] R.Holm, Electric Contacts, Theory and Application, Berlin: Springer-Verlag, $19674^{\text {th }}$ Edition.

[8] M. Nakamura and I. Minowa, "Computer simulation for the conductance of a contact interface," IEEE Trans. Comp., Hybrids, Manufact.Technol., vol. CHMT-9, pp. 150-155, June 1986.

[9] M. Nakamura and I. Minowa, "Film resistance and constriction effect of current in a contact interface," IEEE Trans. Comp., Hybrids, Manufact. Technol., vol.CHMT-12, pp. 109-113, Mar. 1989.

[10] M. Nakamura, "Constriction resistance of conducting spots by the boundary element method," IEEE Trans. Comp., Hybrids, Manufact.Technol., vol. CHMT-16, pp. 339-343, May 1993.

[11] M. Nakamura, "Computer simulation for the constriction resistance depending on the form of conducting spots,” IEEE Trans. Comp., Packag., Manuf.Technol. A, vol. 18, pp. 382-383, June 1995.

[12] J. A. Greenwood, "Constriction resistance and the real area of contact,"Brit. J. Appl. Phys., vol. 17, pp. $1621-1632,1966$.

[13]L. Boyer, "Contact resistance calculations: generalizations of Greenwood's formula including interface films," in IEEE Transactions on Components and Packaging Technologies, vol. 24, no. 1, pp. 50-58, March 2001. 